\title{
Ethical Leadership Connects Voice Behavior: The Mediating Role of Harmony
}

\author{
Manh-Cuong Vu ${ }^{1}$, Quang-Thang Tran ${ }^{2,}$, Thi-Trang-Nhung Nguyen ${ }^{2}$ \\ ${ }^{1}$ Business School, Hunan University, Changsha, China \\ ${ }^{2}$ Department of Business Administration, Hanoi University of Industry, Ha Noi, Viet Nam \\ Email address: \\ Cuongvu3108@gmail.com (Manh-Cuong Vu), Tranquangthang@haui.edu.vn (Quang-Thang Tran), \\ Nguyenthitrangnhung@haui.edu.vn (Thi-Trang-Nhung Nguyen) \\ ${ }^{*}$ Corresponding author
}

\section{To cite this article:}

Manh-Cuong Vu, Quang-Thang Tran, Thi-Trang-Nhung Nguyen. Ethical Leadership Connects Voice Behavior: The Mediating Role of Harmony. Journal of Business and Economic Development. Vol. 5, No. 2, 2020, pp. 56-63. doi: 10.11648/j.jbed.20200502.11

Received: February 24, 2020; Accepted: March 12, 2020; Published: March 24, 2020

\begin{abstract}
This paper presents the findings of a study which examined the relationship between ethical leadership, organizational harmony, and two dimensions of employee voice behavior including employee promotive voice behavior and employee prohibitive voice behavior in the Vietnamese workplace. Further, the study determined the influence of ethical leadership, and organizational harmony on two dimensions of employee voice behavior. This articles also test the mediating role of organizational harmony in the relationship between ethical leadership and employee voice behavior. Although these interrelations are very important for enhancing employee voice behavior, few empirical studies analyze these relationship together. Besides, few empirical studies analyze these relationship in the Vietnamese context. Finally, the study examined the most important factor that influences employee voice behavior. This article explores those relationships using SEM with data from 687 employees in Vietnamese companies. The findings indicated that ethical leaders can foster organizational harmony that promotes employee promotive and prohibitive voice behavior. The analytical results also shown that organizational harmony plays a mediating role in the relationship between ethical leadership and employee promotive and prohibitive voice behavior. On the other hand, there is a positive association exists between organizational harmony and employee promotive and prohibitive voice behavior. Implications for future research, theory, and practice were discussed.
\end{abstract}

Keywords: Ethical Leadership, Organizational Harmony, Promotive Voice, Prohibitive Voice

\section{Introduction}

Voice behavior is becoming one of the hot topics that received much attention from scholars and practitioners, especially in case of Asia firms. Employee voice encourages firms to learn more about their mistakes and weaknesses. The company' services and products are improved the quality and innovation through this $[1,2]$. Moreover, employee voice play an important role in improve involvement models that link HRM and performance [3]. But, employee voice behavior is an action that is pro-social. It is not always naturally occur as expected. In order to increase this proactive behavior, some scholars have an effort to investigate a range of factors that drive voice behavior.
Ethical leadership play a vital role among these factors [4, 5]. Ethical leadership was introduced first by Brown, et al. [4] from a social learning perspective. Ethical leadership is defined as "the demonstration of normatively appropriate conduct through personal actions and interpersonal relationships, and the promotion of such conduct to followers through two-way communication, reinforcement and decision-making" [4, p. 120]. While the social learning approach to understanding ethical leadership holds intuitive and theoretical appeal, most of the existing empirical research focuses on direct effects or uses other theoretical frameworks to explore mediators between ethical leadership and employee voice.

The literature emphasizes the important role that organizational harmony plays on firm's survival and effective 
performance. Therefore, this study has been conducted to explore ethical leadership's impacts on two dimensions of voice behavior (promotive voice and prohibitive voice) through the mediating role of organizational harmony. We expect that the research will contribute new knowledge on how ethical leadership may effect of the concept of voice behavior (Figure 1).

Our study attempts to address the following research questions:

RQ1. Does Ethical leadership influence two dimensions of voice behavior?

RQ2. Does organizational harmony play a mediating role between ethical leadership and two dimensions of voice behavior.

To answer these questions, structural equations modeling is applied to investigate the degree of influence that each variable has on the others, based on the survey of 687 employees in Vietnamese companies. By answering the above questions, the first goal of this study is to analyze and provide empirical evidence about the relationship between Ethical Leadership, aspects of organizational harmony, promotive voice and prohibitive voice. For achievement of the second goal, this study not only estimates direct influences but also explores, more specifically, the indirect impacts of mediating variable on promotive voice and prohibitive voice. Therefore, the article is expected to provide specific and effective solutions for directors or managers in what concerns employee voice.

In order to achieve these objectives, the article is organized in four main sections. First, literature review on ethical leadership, organizational harmony and voice behavior is presented, then the hypotheses were developed. Second, the research methodology is given. Afterwards, the field study analyses were given comparatively with the results described in the literature review above. Finally, the results of the study, strengths and weaknesses, research implications, and future research recommendations are outlined.

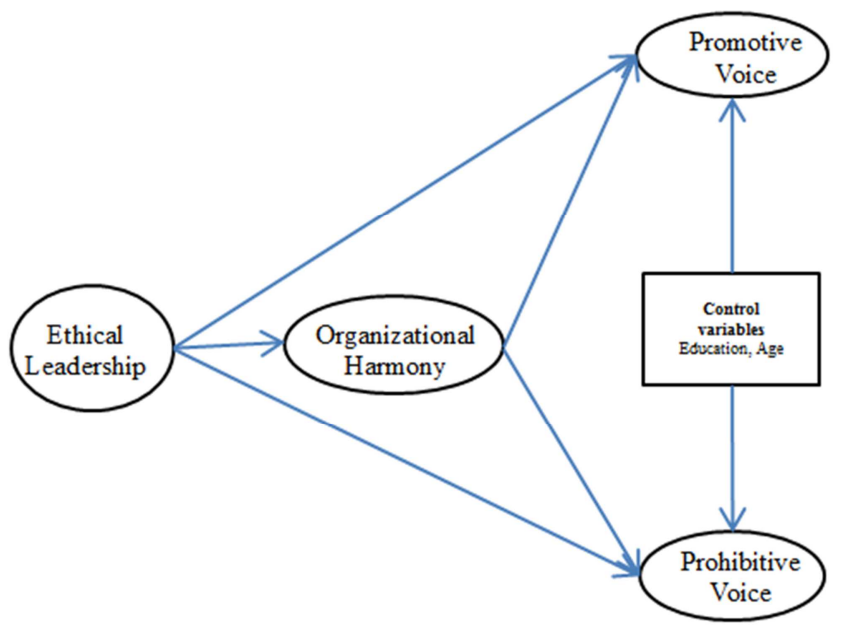

Figure 1. Hypothesized model.

\section{Literature Review and Hypotheses Development}

\subsection{The Effect of Ethical Leadership Behaviors on Employee Voice Behavior}

In the literature review about employee positive behavior, voice behavior has been a hot topic which has grown greatly during the past decades. Voice is primarily motivated by individual desire to highlight dissatisfaction as well as suggest improvements, therefore, voice can be used as an effective means for workers to become more involved in their workplace and to help their organizations to innovate [6].

The concept of voice behavior can be defined as a kind of intentional, speaking-up behavior that be delivered constructive opinions and ideas on work-related issues by employees in organizations to achieve better effectiveness of avoid potential crises [6-8]. However, according to the definition about voice behavior of the United States Department of Labor, employee voice behavior is an employee' ability to access information on their rights in the workplace, their understanding of those rights, and their ability to exercise those rights without fear of discrimination or retaliation. Although there are some the differences when comparing contents between two definitions, the importance of a work environment with ethics and psychological safety to motive employees's voice behavior is identified.

In additions, employee voice behavior is as "promotive behavior that emphasizes expression of constructive challenge intended to improve rather than merely criticize" [9]. Two-dimensional scale for voice behavior is used in this study, namely promotive voice and prohibitive voice [6]. There were some existing research that shown the relationship between ethical leadership and voice behavior [10]. However, the question remains as to how exactly such leadership exerts its effect. Social learning theory indicated that employees observe their leaders' behaviors [4]. The social learning proposes the positive effects of ethical leadership in the following ways: First, leaders impacts on employee ethical behavior through modeling. Leaders give some guidance and convey uninterrupted high moral standards to employees, as well as deep interaction, thereby establishing a longterm trust and reciprocity relationship which benefits the organization. Second, ethical leadership has the relationship with its oen positive characteristics such as honesty, fairness, and trustworthiness). Employees are encouraged to demonstrate moral behaviors and express themselves in the workplace. When employees fell that they in a friendly and fair environment, they tend to demonstrate more prosocial behaviors, including voice behavior.

Promotive voice behavior of employees target on communicating ideal future states for the organization [11]. They focus their efforts on moving the organization closer toward desirable goals. Promotive voice includes a solution that an individuals demonstrate their competence and selfreliance [12]. In the work context, ethical leaders stress the importance of mutual communication in such a way that 
supports employees spesking up [1]. The empirical reseach of Brown, et al. [4] indicated that ethical leadership has a significantly relationship to members' willingness to report issues to their leader. Therefore, employees who perceive high levels of ethical leadership should tend to view promotice voice behavior as desirable and are thus likely to speak up [12].

Prohibitive voice behaviors focus on troublesome and dysfunctional work behaviors. The aim behind prohibitive voice is to pull organizations away from feared states [11]. In the fact that ethical leaders tend to speak out publicly against inappropriate work behaviors and stress doing right things [1]. Ethical leadership improves employees to understand that voicung concerns is valued by the leader and the organization [1]. So, these factors can motivate employees to exhibit prohibitive vocie.

Thus, we hypothesize:

Hypothesis 1a. Ethical leadership, as perceived by employees, is positively related to promotive voice.

Hypotheis 1b. Ethical leadership, as perceived by employees, is positively related to prohibitive voice.

\subsection{Organizational Harmony Mediates the Relationship Between Ethical Leadership and Employee Voice Behavior}

An ethical leader should be responsible for creating the right condition, namely, the suitable organizational soil or culture/climate to nourish the moral development of followers in an organization. In other word, ethical leadership appears to be a key determinant for constituting ethical business culture in enterprises. According to Ardichvili and Jondle [13], the concept of ethical corporate culture can be described as a type of organizational culture based on "an alignment between formal structures, processes and policies, consistent ethical behavior of top leadership, and informal recognition of heroes, stories, rituals, and language that inspire organizational members to behave in a manner accorded with high ethical standards that have been set by executive leadership".

This article aims to address whether ethical leaders can improve employee voice behavior through mediating mechanism of cultural variables such as employee-perceived harmony at workplace. On the premise that ethical leadership is positively and significantly associated with the level of harmony and employee voice behavior, it can be further expected that the mediation effect of harmony on the relationship between ethical leadership and employee voice behavior also exists.

Thus, we hypothesize:

Hypothesis 2. Ethical leadership, as perceived by employees, is positive related to the level of organizational harmony an employee perceives

Hypothesis 3a. Organizational harmony is positively related to promotive voice

Hypothesis 3b. Organizational harmony is positive related to prohibitive voice

Hypothesis 4a. Organizational harmony acts a mediator between ethical leadership and promotive voice

Hypothesis 4b. Organizational harmony acts a mediator between ethical leadership and prohibitive voice

\section{Research Methodology}

\subsection{Sample and Data Collection}

We adopted an empirical data based on a survey of 50 service firms in Vietnam. We first communicated with representatives of these firms by phone. In some cases, we made personal visits to interpret the goals of the research and inquired for the assistance in collecting data. Employees at some important departments of these firms (such as: operation, accounting, R\&D, marketing, or sale) were collected to answer survey questionnaires. This study distributed 900 questionnaires and received 724 ones in final, among which 687 ones were valid $(76.3 \%$ valid rate). Total of 687 respondents, 321 (46.7\%) were male and 366 (53.3\%) were female. They answered the issues relating to the study variables, including: Ethical leadership; organizational harmony, promotive voice, and prohibitive voice in their firm.

\subsection{Variable Measurement}

All the applied scales in this research had been previously published and validated. Scales that were origially formulated in English were translated to Vietnamese and then back to English. All the scales presented satisfactory reliabilities. The means, standard deviations, zero-order correlations, and reliability coefficients are shown in Table 1.

As shown in Table 1, promotive voice was significantly correlated with education, ethical leadership and organizational harmony. Prohibitive voice, on the other hand, was significantly correlated with ethical leadership and organizational harmony.

Table 1. Means, Standard Deviation, and Correlations $(n=687)$.

\begin{tabular}{llllllll}
\hline & M & SD & 1 & 2 & 3 & 6 \\
\hline 1. Education & 2.44 & 0.89 & 1 & & & \\
2. Age & 2.37 & 0.87 & $0.531^{* *}$ & 1 & & \\
3. EthLead & 3.94 & 0.60 & $0.395^{* *}$ & $0.280^{* *}$ & 1 & \\
4. OrHar & 3.73 & 0.58 & $0.394^{* *}$ & $0.392^{* *}$ & $0.426^{* *}$ & 1 \\
5. PromVoi & 3.74 & 0.67 & $0.595^{* *}$ & $0.658^{* *}$ & $0.335^{* *}$ & $0.468^{* *}$ \\
6. ProhVoi & 3.80 & 0.60 & $0.313^{* *}$ & $0.280^{* *}$ & $0.536^{* *}$ & $0.491^{* *}$ & $0.337^{* *}$ \\
\hline
\end{tabular}

Note: EthLead=Ethical Leadership, OrHar=Organizational Harmony, PromVoi=Promotive Voice, ProhVoi=Prohibitive Voice ${ }^{* *}<0.01$. 
Ethical leadership: We measured ethical leadership using a 10-item scale developed by [4]. These items were also adopted in the studies of [14]. A sample item is "My boss established a model of doing things based on code of ethics". Responses were made using a 5-point Likert-type scale (from $1=$ not at all to $5=$ frequently, if not always).

Promotive voice and Prohibitive voice behavior. Promotive and prohibitive voice behavior were measured using a 10-item scale developed by [15], with five items to measure each construct. Responses were made using a 5-point Likert-type scale (from $1=$ strongly disagree to $5=$ strongly agree). This scale seized the diverrgent aspects of promotive and prohibitive voice behavior. We changed slightly the wording to make it suitable for self-reporting. Sample items are "I suggest new projects which may be beneficial to the work unit" (promotive voice) and "I speak up honestly with problems that might cause serious loss to the work unit, even when/though dissenting opinions exist" (prohibitive voice).

Organizational Harmony: We measured organizational harmony using a 32-item scale developed by [16]. This scale includes 8 dimensions. Four items for harmony of individual worker with himself, four for harmony of co-workers, four for harmony of individual worker with his team, four for harmony of employee with his direct supervisor, four for harmony of individual worker with the managerial system, four for harmony between departments, four for harmony between individual worker with the corporate leader, and four for harmony between people inside and outside organizations. [17] suggested to reduce parameters by averaging the eight sub constructs of the model of harmony to form a composite score indicator representing the level of harmony. Thus, we used eight items for organizational harmony in this research.

Control variables: We also included individual demographic chareateristics in the analysis bacause these variables may affect the relationship of interest (e.g., [18]. We add gender, age, tenure and education were used as control variables to account for differences among employees that have potential impacts on employee voice behavior. However, gender and tenure controls were not significantly related to the dependent variables. Since we felt that including nonsignificant control variables would erode degrees of freedom [19], we finally did not control for these two variables. We controlled for the education and age variable because it had been found to be significantly related to voice behavior.

\subsection{Data Analysis Methods}

Analysis of Moment Structures (AMOS) was used for measurement validation and for examining the structural model based on the data gathered form the 687 respondents of 50 service firms. Data analysis was conducted by using SPSS and AMOS version 22.0. Confirmatory factor analysis (CFA) was implemented to test the validity and reliability of the constructs.

\section{Data Analysis and Results}

\subsection{Measurement Model}

We first examined the reliability of the measures of the constructs by testing the individual Cronbach's alpha coefficients through using SPSS version 22.0. The results were that all of them ranged from 0.879 to 0.922 and were all higher than the recommended level of 0.7 [20]. We then used AMOS version 22.0 to conduct a series of confirmatory factor analyses (CFAs) to examine whether the selected variables captured distinct constructs. The results presented in Table 2 show that the four-factor measurement model fit the data well: $\chi^{2}=759.21$, d $f=344, \mathrm{CFI}=0.91, \mathrm{GFI}=0.90, \mathrm{IFI}=0.91, \mathrm{NFI}=0.90$. In addition, all indicators had significant factor loadings $(p<0.01)$, suggesting convergent validity [21]. Besides, this research employed some methods to examine discriminant validity. The one-factor measurement model fit the data poorly: $\chi^{2}=6177.507, \quad$ dl $f=350, \quad \mathrm{CFI}=0.53, \quad \mathrm{GFI}=0.46, \quad \mathrm{IFI}=0.53$, $\mathrm{NFI}=0.52$. The chi-squared difference compared with the fourfactor model was significant $\left(\Delta \chi^{2}=5418.3, \mathrm{p}<0.01\right)$, indicating distinctly different factors. Note that the measures of ethical leadership, organizational harmony, promotive voice, and prohibitive voice were all collected from the same source (employees). This paper used two and three randomly created parcels of items for ethical leadership, organizational harmony, promotive voice, and prohibitive voice to further examine whether these variables captured distinct constructs. The results were that the four-factor measurement model was better than any alternative two-factor measurement and three-factor measurement model. These results supported the discriminant validity of these four measures.

Table 2. Measurement model comparisons.

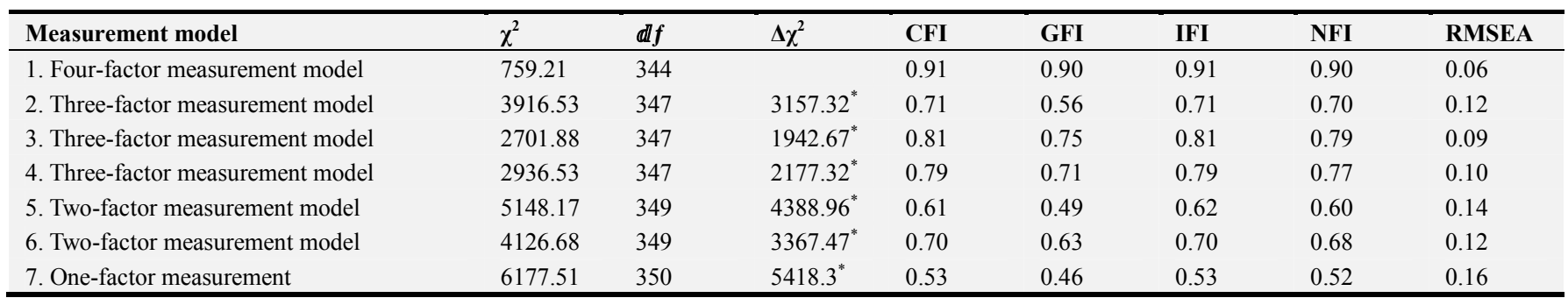

Note: $\mathrm{n}=687$. Model 2 merges ethical leadership and organizational harmony; model 3 merges organizational harmony and promotive voice; model 4 merges promotive voice and prohibitive voice; model 5 merges ethical leadership, organizational harmony and promotive voice; model 6 merges organizational harmony, promotive voice and prohibitive voice; and model 7 merges all variables (ethical leadership, organizational harmony, promotive voice, and prohibitive voice). The $\Delta \chi^{2}$ is in relation to Model $1 .{ }^{*} \mathrm{p}<0.01$. 


\subsection{Structural Model}

This section shown the main results of the hypothesis examining of the structural relationships among the latent variables.

\subsubsection{Direct Effects Analysis}

Multiple regression analyses were performed seperately, considering ethical leadership as the independent variable and organizational harmony and the two dimensions of employee voice behavior as the dependent variables (See table 3). The results in table 3 shown that all the path coefficients of direct effects are found to be significanr and in line with the stated hypothesis. Specifically:

Models 1 and 2 indicated thet ethical leadership is positively associated with promotive voice $(\beta=0.379 ; p<0.05)$ and prohibitive voice $(\beta=0.479 ; \mathrm{p}<0.001)$. Thus, H1a and $H 2 b$ are supported.
Model 3 shown that ethical leadership is positively related to organizational harmony $(\beta=0.302 ; \mathrm{p}<0.001)$. Therefore, $H 3$ is supported.

Models 4 and 5 indicated that organizational harmony is positively related to both promotive voice $(\beta=0.183$; $\mathrm{p}<0.001)$ and prohibitive voice $(\beta=0.424 ; \mathrm{p}<0.001) . H 3 a$ and $H 3 a$ are also supported.

We examined the control role of education and age for ethical leadership over 4 models (the whole models 1-5, excepting model 3). The results indicated that the influence of education and age on promotive voice and prohibitive voice are significant at $p$-value more than 0.05 in all models. It implied that employee with higher education will have greater potential to promotive and prohibitive voice. Similarly, employee with higher age will have greater potential to promotive and prohibitive voice.

Table 3. The effects of ethical leadership on organizational harmony and employee voice behavior.

\begin{tabular}{llllll}
\hline \multirow{2}{*}{ Variable } & \multicolumn{2}{l}{ Voice Behavior } & & \multirow{2}{*}{ OrHar } & \multicolumn{2}{c}{ Voice Behavior } \\
\cline { 2 - 3 } & PromVoi & ProhVoi & PromVoi & ProhVoi \\
\cline { 2 - 3 } & Model 1 & Model 2 & Model 3 & Model 4 & Model 5 \\
\hline Control variable & & & & \\
Education & $0.315^{* * *}$ & $0.065^{* * *}$ & $0.155^{* *}$ & $0.295^{* * *}$ & $0.119^{* * *}$ \\
Age & $0.269^{* * *}$ & $0.111^{* *}$ & $0.225^{* *}$ & $0.430^{* * *}$ & $0.050^{* * *}$ \\
Independent variable & & & & \\
Ethical leadership & $0.379^{* *}$ & $0.479^{* * *}$ & $0.302^{* * *}$ & & $0.424^{* * *}$ \\
OrHar & & & & $0.183^{* * *}$ & 0.259 \\
$\mathrm{R}^{2}$ & 0.432 & 0.308 & 0.278 & 0.544 & 0.256 \\
Adjusted $\mathrm{R}^{2}$ & 0.427 & 0.305 & 0.275 & 0.542 & $79.774^{* * *}$ \\
$\mathrm{~F}$ & $249.046^{* * *}$ & $101.235^{* * *}$ & $87.683^{* * *}$ & $271.558^{* * *}$ & \\
\hline
\end{tabular}

Notes: ${ }^{* * *} \mathrm{p}<0.001 ;{ }^{* *} \mathrm{p}<0.05 ; \mathrm{n}=687$; OrHar: Organizational Harmony; PromVoi: Promotive Voice; ProhVoi: Prohibitive Voice.

\subsubsection{Test of the Mediating Effect}

Models 6 and 7 in table 4 and figure 2 shown that after organizational harmony has been added as a mediator between ethical leadership and promotive voice (model 6), and between ethical leadership and prohibitive voice (model 7), organizational harmony's effects on promotive voice $(\beta=0.175$; $\mathrm{p}<0.001)$ and prohibitive voice $(\beta=0.301 ; \mathrm{p}<0.001)$ are significant. However, for ethical leadership's effects, as compared with models 1 and 2, the direct effect of ethical leadership on promotive voice decreases from $0.379(p<0.05)$ to $0.326(p<0.001)$ and ethical leadership's effects on prohibitve voice decreases from $0.479(\mathrm{p}<0.001)$ to $0.388(\mathrm{p}<0.001)$. Therefore, organizational harmony partially mediates the effects of ethical leadership on two dimensions of voice behavior.

Table 4. Test of mediating effects.

\begin{tabular}{lll}
\hline \multirow{2}{*}{ Variable } & \multicolumn{2}{l}{ Mediating effect } \\
\cline { 2 - 3 } & PromVoi & ProhVoi \\
\cline { 2 - 3 } & Model 6 & Model 7 \\
\hline Control variable & $0.288^{* * *}$ & $0.018^{* * *}$ \\
Education & $0.230^{* * *}$ & $0.044^{* * *}$ \\
Age & & \\
Independent variable & $0.326^{* *}$ & $0.388^{* * *}$ \\
Ethical leadership & & \\
Mediators & $0.175^{* * *}$ & $0.301^{* * *}$ \\
OrHar & 0.544 & 0.373 \\
$\mathrm{R}^{2}$ & 0.542 & 0.370 \\
Adjusted $\mathrm{R}^{2}$ & $203.799^{* * *}$ & $101.588^{* * *}$ \\
$\mathrm{~F}$ & & \\
\hline
\end{tabular}




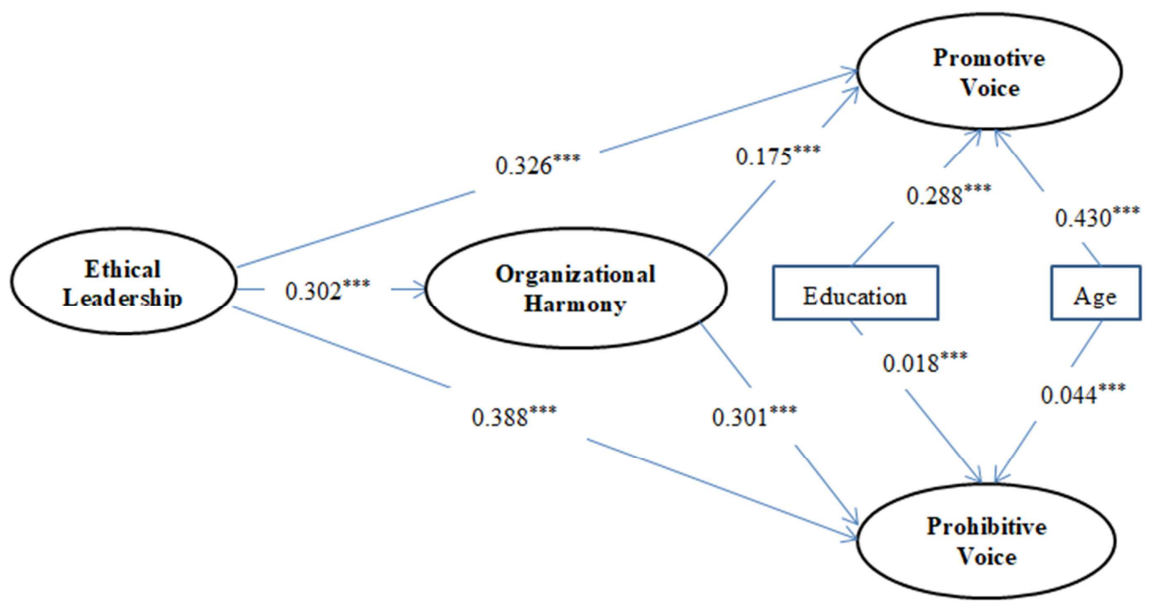

Figure 2. Path diagram of the mediating model.

Besides, This research implemented further analyses to verify the magnitude and the statistical significance of the indirect effects. We used the bootstrap confidence intervals method with 4000 iterations to examine the significance of indirect effects based on the suggestion of Preacher and Hayes [22] about statistical inferences (See table 4).

Table 5 presented that the indirect effects of ethical leadership on promotive voice $(\beta=0.322 ; \mathrm{p}<0.001)$ and prohibitive voice $(\beta=0.357 ; \mathrm{p}<0.001)$ are significant within the range of confidence intervals. Hence, hypotheses $\mathrm{H} 4 \mathrm{a}$ and $H 4 b$ are supported. In general, these findings are the first to confirm the mediating role of organizational harmony in the relationship between ethical leadership and employee voice behavior.

Table 5. Confidence intervals of the indirect effects.

\begin{tabular}{lllll}
\hline Path & Direct effects & Indirect effects & Total effects & \multicolumn{2}{c}{ Bias-corrected confidence intervals } & Lower confidence level & Upper confidence level \\
\hline EthLead--> OrHar--> PromVoi & $0.326^{* * *}$ & $0.322^{* * *}$ & $0.348^{* * *}$ & 0.298 \\
EthLead--> OrHar--> ProhVoi & $0.388^{* * *}$ & $0.357^{* * *}$ & $0.745^{* * *}$ & 0.301 \\
\hline
\end{tabular}

Notes: ${ }^{* * *}$ p $<0.001$; EthLead: Ethical Leadership; OrHar: Organizational Harmony; PromVoice: Promotive Voice; ProhVoi: Prohibitive Voice.

\section{Discussion}

Employee Voice has achieved widespread popularity as a high level of performance management approach that allows organizations to sustain competitive and innovative. This paper explored the effectiveness of ethical leadership on encouraging promotive and prohibitive voice behavior among employees in Vietnamese Firms.

The empirical results of this paper support to our hypotheses. Firsts, the findings suggest that employee-rated ethical leadership has positive relationship with employeeperceived harmony in eight dimensions. Second, there are a significant relationship between the employee-rated ethical leadership and two dimensions of employee voice behavior (promotive voice and prohibitive voice). Third, results are consistent with the predicted mediation model that the level of harmony positively mediates the relationship between employee-perceived harmony and two dimensions of employee voice behavior.

This paper supports some main contributions. First, given research on ethical leadership is still in its early stages of development [23], a main implication of this study is to explain the impotant role of ethical leadership in management practices in the complex yet dynamic Vietnamese context. The findings contribute on the leadership literature by testing the impact of ethical leadership on employee voice behavior via enhancing organizational harmony in Vietnam. Second, thí paper contributes the current HRM literature. Given the existing Western theories that currently dominate the HRM literature may not be always adequate to meet the need of workers in Vietnam due to the difference of culture, this paper, thus, is in response to the recent appeal for enhancing context-sensitive indigenous theories and analyzing country-specific phenomena. Third, this paper allows other firms that be not in Vietnam can achieve a better understanding of the positive effect of ethical leadership on the level of harmony in Vietnamese organizations. Ours findings suggest that facing highly uncertain business environment in Vietnam, managers could put more effort into gain the higher level of ethical behaviors to enhance a harmonious leader-follower relationship and through which to encourage employees to speak up for promoting organizational health.

\section{Limitations and Directions for Future Research}

The article had provided the understanding and values to the literature and practice, it has certain limitations. First, because employees provided ratings of ethical leadership, organizational harmony, two dimensions of employee voice, 
the hypothesized relationships between ethical leadership and the mediating varible must be interpreted with caution due to same-source concerns. For example, it is possible that employees' rating of ethical leadership biased their ratings of perceptions of organizational harmony and two dimensions of employee voice behavior. Future research should strike to measure all predictors from different sources or utilize manipulations or objective outcomes.

Second, although we did test the theoretically relevant mediators and examine their effects simultaneously, other mechanisms could help explain the relationship between ethical leadership and employee voice. For example, [Yuan, et al. [14]] found that leader-member exchange mediated this relationship. Future research should provide a more exhaustive test of different mediators including leadermember exchange, the mediators we assessed, as well as other potential mediators or moderators.

\section{Conclusions}

This article's findings provided significant theoretical and practical implications for literature on leader's behavior, organizational management, and employee behavior that can be used to analyze the links among ethical leadership, organizational harmony and employee voice behavior. The findings verify the hypotheses that ethical leadership and organizational harmony have positive and significant roles in promoting. Overall, by operating a climate of harmony among employees, leaders can create a positive and appropriate environment to facilitate individual attitudes and significantly contribute to enhancing promotive and prohibitive voice behavior for their employees.

\section{References}

[1] J. B. Avey, T. S. Wernsing, and M. E. Palanski, "Exploring the process of ethical leadership: The mediating role of employee voice and psychological ownership," Journal of Business Ethics, vol. 107, pp. 21-34, 2012.

[2] X. Zhou, J.-Q. Liao, Y. Liu, and S. Liao, "Leader impression management and employee voice behavior: Trust and suspicion as mediators," Social Behavior and Personality: an international journal, vol. 45, pp. 1843-1854, 2017.

[3] B. Kwon, E. Farndale, and J. G. Park, "Employee voice and work engagement: Macro, meso, and micro-level drivers of convergence?," Human Resource Management Review, vol. 26, pp. 327-337, 2016.

[4] M. E. Brown, L. K. Treviño, and D. A. Harrison, "Ethical leadership: A social learning perspective for construct development and testing," Organizational behavior and human decision processes, vol. 97, pp. 117-134, 2005.

[5] T. W. Ng and D. C. Feldman, "Employee voice behavior: A meta-analytic test of the conservation of resources framework," Journal of Organizational Behavior, vol. 33, pp. 216-234, 2012.

[6] J. Liang, C. I. Farh, and J.-L. Farh, "Psychological antecedents of promotive and prohibitive voice: A two-wave examination," Academy of Management Journal, vol. 55, pp. 71-92, 2012.

[7] V. Venkataramani and S. Tangirala, "When and why do central employees speak up? An examination of mediating and moderating variables," Journal of Applied Psychology, vol. 95, p. 582, 2010.

[8] G. Gazzoli, A. Zablah, and T. Brown, "Why do frontline employee speak up on behalf of customers? The influence of supervisors vesus coworkers and the role of intrapersonal factors," in Robert Mittelsaedt Doctoral Symposium, 2017, p. 217.

[9] L. Van Dyne and J. A. LePine, "Helping and voice extra-role behaviors: Evidence of construct and predictive validity," Academy of Management journal, vol. 41, pp. 108-119, 1998.

[10] A. S.-Y. Chen and Y.-H. Hou, "The effects of ethical leadership, voice behavior and climates for innovation on creativity: A moderated mediation examination," The Leadership Quarterly, vol. 27, pp. 1-13, 2016.

[11] S.-H. J. Lin and R. E. Johnson, "A suggestion to improve a day keeps your depletion away: Examining promotive and prohibitive voice behaviors within a regulatory focus and ego depletion framework," Journal of Applied Psychology, vol. 100, p. 1381, 2015.

[12] X. Wei, Z.-X. Zhang, and X.-P. Chen, "I will speak up if my voice is socially desirable: A moderated mediating process of promotive versus prohibitive voice," Journal of Applied Psychology, vol. 100, p. 1641, 2015.

[13] A. Ardichvili and D. Jondle, "Integrative literature review: Ethical business cultures: A literature review and implications for HRD," Human Resource Development Review, vol. 8, pp. 223-244, 2009.

[14] L. Yuan, M.-C. Vu, and T.-T.-N. Nguyen, "Ethical leadership, leader-member exchange and voice behavior: Test of mediation and moderation processes," in Proceedings of the 2018 2nd International Conference on Management Engineering, Software Engineering and Service Sciences, 2018, pp. 33-42.

[15] J. Liang, C. I. C. Farh, and J.-L. Farh, "Psychological antecedents of promotive and prohibitive voice: a two-wave examination," The Academy of Management Journal, vol. 55, pp. 71-92, 2012.

[16] T. Chin, "An empirical study on harmonious organizations," Journal of Sun Yat-sen University, vol. 50, pp. 164-174, 2010.

[17] L. Zhou, B. R. Barnes, and Y. Lu, "Entrepreneurial proclivity, capability upgrading and performance advantage of newness among international new ventures," Journal of International Business Studies, vol. 41, pp. 882-905, 2010.

[18] M. E. Debus, T. M. Probst, C. J. König, and M. Kleinmann, "Catch me if I fall! Enacted uncertainty avoidance and the social safety net as country-level moderators in the job insecurity-job attitudes link," Journal of Applied Psychology, vol. 97, p. 690, 2012.

[19] G. Atinc, M. J. Simmering, and M. J. Kroll, "Control variable use and reporting in macro and micro management research," Organizational Research Methods, vol. 15, pp. 57-74, 2012.

[20] J. C. Nunnally, Psychometric theory 3E: Tata McGraw-Hill Education, 1994. 
[21] J. C. Anderson and D. W. Gerbing, "Structural equation modeling in practice: A review and recommended two-step approach," Psychological bulletin, vol. 103, p. 411, 1988.

[22] K. J. Preacher and A. F. Hayes, "Asymptotic and resampling strategies for assessing and comparing indirect effects in multiple mediator models," Behavior research methods, vol. 40, pp. 879-891, 2008.
[23] D. M. Mayer, K. Aquino, R. L. Greenbaum, and M. Kuenzi, "Who displays ethical leadership, and why does it matter? An examination of antecedents and consequences of ethical leadership," Academy of Management Journal, vol. 55, pp. 151-171, 2012. 